\title{
Simulation Research on Acoustic Detection Technology of Buried PE Pipes
}

\author{
Bo Chen ${ }^{1,2}$, Jiao Lan ${ }^{1}$, Liang $\mathrm{Ge}^{1,2^{*}}$, Yunfeng $\mathrm{Lu}^{1}$, Yingbo $\mathrm{Hu}^{1}$, Wenhai $\mathrm{Li}^{1}$, Cui Lai ${ }^{1}$, \\ Xiaoting Xiao ${ }^{1,2}$, Qi Huang ${ }^{1}$ \\ ${ }^{1}$ Southwest Petroleum University, Chengdu, 610500, China \\ ${ }^{2}$ The National Mountain Highway Engineering Technology Research Center, Chongqing, \\ 400074, China
}

Received: October 17, 2020. Revised: April 7, 2021. Accepted: April 18, 2021. Published: April 23, 2021.

\begin{abstract}
Polyethylene (PE) pipe has been widely
\end{abstract} used in gas transportation pipeline system because it overcomes the shortcomings of non-corrosion resistance of metal pipelines. However, the stiffness and strength of PE pipe are small, and it is easy to be destroyed in the third-party construction process. Moreover, PE pipe is not electrically or magnetically conductive, and it is unable to use the developed metal pipe detection method, which has brought great security risks. Urban gas pipeline accidents occur frequently, and the situation during the production is complex and severe. Therefore, it is of great significance to study how to effectively realize the detection of underground $P E$ pipe. This paper verifies the feasibility of the acoustic method from the perspective of simulation. Firstly, it studies the influence of buried pipe depth on the received signal of geophone. Secondly, it studies the influence of buried pipe size on the received signal of $\mathrm{PE}$ geophone with a certain depth Finally, it studies the received signal of geophone when PE pipe is in mixed soil. The simulation results show that the method based on acoustic wave is suitable, the signal source emits a sweeping frequency single tone sine wave, which propagates to the target and is reflected. A string of geophones on the ground measure the velocity of the surface vibration. Given the propagation rate of wave in the material, time delay can be used between signal sent and received on the ground to obtain the estimated distance to the target.
Keywords: buried polyethylene Pipe Detection; Acoustic Simulation; COMSOL Multiphysics

\section{INTRODUCTION}

$\mathrm{n}$ view of the special properties of $\mathrm{PE}$ pipe, such as
non-conductive and non-magnetic, due to the low strength of PE pipe, the marking and tracing line are often destroyed, and the site construction drawings are easily lost. In the construction process, due to the unclear exact position of $\mathrm{PE}$ pipe, it is often cut by construction machinery, resulting in accidents such as gas leakage and explosion and water leakage loss of water pipe. Therefore, how to realize the positioning detection of urban buried PE pipeline is very important. According to the different positioning principle, the underground PE pipe positioning method can be divided into conventional buried PE pipe detection method and new buried PE pipe detection method [1]. Conventional buried PE pipe detection methods include tracer line detection method, geological radar detection method, pipeline acoustic positioning method and static electromagnetic field positioning method. The new buried PE pipe detection methods include underground electronic identification system, geographic information system (GIS), intelligent robot tracking and positioning, harmonic radar (frequency doubling) radio frequency tag [2].

Tracer line detection is a common method at home and 
abroad. This method follows the traditional metal pipe positioning principle, namely electromagnetic induction principle. During the detection, a metal wire is laid on the surface of the PE pipe to overcome the shortcomings of non-conductive and non-magnetic PE pipe. The metal wire has obvious dew point at the valve for external transmitter. Then the ground detector can detect different signals according to the electromagnetic field generated by the metal wire, which can determine the location, depth and direction of the PE pipeline. Geological radar detection is a non-destructive geophysical exploration method using high frequency electromagnetic wave to detect the distribution of underground medium. This method mainly uses electromagnetic wave reflected by underground medium to detect the position information of PE pipe [3]. Using the electromagnetic wave reflected by the receiver, the time, phase, amplitude, wavelength and other characteristics of the reflected wave arrival are accurately recorded by the radar host, and then the scanning image of the underground section is formed by data processing methods such as signal superposition and amplification, filtering and noise reduction, and image synthesis. Through the interpretation of radar images, the distribution position and state of underground targets can be obtained. Static electromagnetic field positioning method uses the transmitter to transmit different frequency signals to different underground impurities. When this signal encounters impurities in the process of underground propagation, the signal will also change due to the change of propagation medium, and the surface receiving antenna will change its antenna state due to the change of receiving signal. According to the change of antenna, the presence, location, direction and size of buried impurities can be judged.

At present, the location detection methods of buried PE pipelines have their own characteristics, but each method has some limitations, and it does not have the ability to detect PE pipelines in any environment or the effect is not ideal. How to comprehensively utilize the current technology to accurately locate the buried PE pipeline is particularly important. Compared with other similar products, acoustic detection method has less limited conditions. Acoustic detection method is preferred in the absence of tracer lines or incomplete tracer lines. Acoustic positioning detection technology does not need to connect with the pipeline to accurately locate. It can realize nondestructive safety detection of underground PE pipelines, especially in urban underground pipelines. The advantages are more obvious when there are more interference sources. At the same time, pipeline acoustic positioning method is not limited by the environment. Asphalt pavement, ceramic tile pavement, soil pavement, gravel pavement, concrete pavement and other complex ground conditions can be used to detect under various conditions.

According to the technical principles and characteristics of these two kinds of buried PE pipeline positioning methods. It is of great significance and practical significance to establish a more perfect and intelligent underground PE pipeline detection system, the innovation of buried PE pipeline detection principle, and the improvement of existing buried PE pipeline detection technology. Acoustic positioning technology applied to buried pipe positioning detection is a new technology, which mainly uses the propagation characteristics of sound waves to detect and locate targets, where the detection wave amplitude corresponds to the detection time or distance [4], [5]. In the pipeline acoustic positioning detection, an emitter is used to generate a short sound wave pulse to the ground, and the ground surface wave is then back-reflected by the discontinuous interface or interface with unmatched sound wave impedance [6]. The reflection coefficients of the smooth pipeline internal surface and gas interface are 100\% while that of the interface between rock or soil and the pipeline external surface is relatively lower. Then the receiver is used to receive ground surface wave and reflected wave, and the oscilloscope is employed to observe the two waves for the target detection and location.

The finite element analysis method realizes the simulation of real physical phenomena by solving partial differential equations (single field) or partial differential equations (multi field). The mathematical method is used to solve the physical phenomena in the real world. COMSOL Multiphysics realizes highly accurate numerical simulation with efficient calculation performance and outstanding multi field bidirectional direct coupling analysis ability. The predefined multi-physical field application mode can solve many common physical problems. COMSOL Multiphysics has the characteristics of wide use, flexibility and ease of use. Compared with other finite element analysis software, 
COMSOL Multiphysics has the advantage of using additional functional modules, which can be easily extended.

In the second section, the refraction and reflection theory at the junction of PE pipe is introduced. In the third section, the establishment process of finite element simulation model based on COMSOL Multiphysics is described in detail [7]. In the fourth section, the simulation results are analyzed in detail and summarized.

\section{REFLECTION THEROY OF ELASTIC WAVE IN SOIL MEDIUM}

Under normal depth, positioning pipeline detection accuracy is high. If the depth is less than $2.4 \mathrm{~m}$, the pipeline with diameter more than $100 \mathrm{~mm}$ can be located; when the depth is less than $1.2 \mathrm{~m}$, the pipeline with diameter above 51 $\mathrm{mm}$ can be located; When the depth is less than $0.8 \mathrm{~m}$, the pipeline with diameter above $13 \mathrm{~mm}$ can be located.

In the actual soil, the situation is more complex. There are many uncertain factors in the wave propagation. It is obviously unreasonable to specify the soil as the ideal condition. However, this paper mainly focuses on the refraction and reflection of wave at the junction of soil and PE pipe and its simulation, so the nonlinear factors of soil and wave are not discussed. In the following studies, it is assumed that the soil is in ideal condition, and the wave also propagates in the soil as elastic wave. Firstly, the wave of elastic wave in ideal medium is analyzed.

There are $\mathrm{P}$-wave and SV-wave in the uniform infinite medium. When the elastic wave propagates in the isotropic medium, the elastic wave will propagate in the isotropic medium [8]. Lame equation can be used to describe the differential equation of displacement:

$$
\rho \frac{\partial^{2} \vec{U}}{\partial \mathrm{t}^{2}}=(\lambda+\mu) \operatorname{grad} \theta+\mu \nabla^{2} \vec{U}+\rho \vec{F}
$$

Where, $\vec{U}$ is the displacement vector, $\vec{F}$ is external force vector, $\theta=\operatorname{div} \vec{U}$ is the volume strain, i.e. the field shift divergence, $\quad \rho$ is the density of the medium, which is constant, $\lambda, \mu$ is lame coefficient, $\nabla^{2}$ is Laplacian operator, $\nabla^{2}=\frac{\partial^{2}}{\partial x^{2}}+\frac{\partial^{2}}{\partial y^{2}}+\frac{\partial^{2}}{\partial z^{2}}$, grad is Gradient operator, $\nabla=\frac{\partial}{\partial \mathrm{x}} \vec{i}+\frac{\partial}{\partial y} \overrightarrow{\mathrm{j}}+\frac{\partial}{\partial z} \vec{k}$

Equation (1) is a differential equation of motion expressed by vector. Taking divergence and curl on both sides of the formula, we can get the following results.

$$
\begin{aligned}
& \rho \frac{\partial^{2} \theta}{\partial \mathrm{t}^{2}}-\frac{(\lambda+2 \mu)}{\rho} \nabla^{2} \theta=\operatorname{div} \vec{F} \\
& \frac{\partial^{2} \vec{\omega}}{\partial \mathrm{t}^{2}}-\frac{\mu}{\rho} \nabla^{2} \vec{\omega}=\operatorname{rot} \vec{F}
\end{aligned}
$$

Where, $\vec{\omega}=\operatorname{rot} \vec{U} ; \operatorname{div} \vec{F}$ is expansion and contraction

force, $\operatorname{rot} \vec{F}$ is a kind of rotating force. Formula (2) describes that under the action of expansion and contraction force $\operatorname{div} \vec{F}$, the medium only produces volume related disturbance, which is called longitudinal wave. Formula (3) describes the deformation disturbance determined by the rotation vector $\vec{\omega}=\operatorname{rot} \vec{U}$ produced by the medium under the action of rotating external force $\operatorname{rot} \vec{F}$, which is called shear wave.

According to Helmholtz theorem, the vector field can be expressed by the sum of a scalar gradient field and the curl field of a vector bit. Where,

$$
\begin{aligned}
& \vec{U}=u_{p}+u_{s}=\operatorname{grad} \phi+\operatorname{rot} \vec{\psi} \\
& \vec{F}=F_{p}+F_{s}=\operatorname{grad} \Phi+\operatorname{rot} \vec{\Psi}
\end{aligned}
$$

In the above formula, $\phi$ and $\vec{\psi}$ represent scalar potential and vector potential of displacement field $\vec{U}$ respectively. $\Phi$ and $\vec{\Psi}$ represent scalar potential and vector potential of external force field $\vec{F}$ respectively.

Note $\theta=\operatorname{div} \vec{U}=\frac{\partial u}{\partial x}+\frac{\partial v}{\partial y}+\frac{\partial \omega}{\partial z}, \vec{\omega}=\operatorname{rot} \vec{U}$ and the wave equation expressed by shift function without considering external force is the following formula.

$$
\begin{aligned}
& \frac{\partial^{2} \phi}{\partial t^{2}}-v_{p}^{2} \nabla^{2} \phi=0 \\
& \frac{\partial^{2} \vec{\psi}}{\partial t^{2}}-v_{s}^{2} \nabla^{2} \vec{\psi}=0
\end{aligned}
$$

Where $v_{p}^{2}=\frac{\lambda+2 \mu}{\rho}$ and $v_{s}^{2}=\frac{\lambda}{\rho} \quad$ represent the propagation velocity of $\mathrm{P}$-wave and $\mathrm{SV}$-wave respectively.

In the actual measurement, the wave propagation is prone to the disturbance of environmental factors and the 
instability. But in ideal case, the displacement of elastic wave at different media junction can be recognized by the derivation of scalar and vector bits of the displacement field. Comprehensive analysis on the propagation characteristics of the elastic wave in the soil combining the displacement equations has important value and verification significance for the following theoretical deduction of the acoustic positioning technology.

\section{III. REFLECTION THEORY OF ELASTIC WAVE AT}

\section{SOIL INTERFACE}

Only P-wave radiation from the source to homogeneous media is taken into consideration here. Refraction and reflection of the $\mathrm{P}$ wave occur at the interface. The propagation velocity of the incident wave in each medium is constant, and the mode conversion of the incident wave at the interface also meets the Snell ' $s$ law. It is assumed that the mode conversion waves generated by the incident wave propagate in the $x z$ plane. The incident angle of the incident $\mathrm{P}$-wave is $\theta^{(0)}$, and the wave number is $k^{(0)}$. The reflection angle of the reflected $\mathrm{P}$-wave is $\theta^{(1)}$, and the wave number is $k^{(1)}$. The reflection angle of the reflected SV-wave is $\theta^{(3)}$, and the wave number is $k^{(3)}$. The refraction angle of the refracted $\mathrm{P}$-wave is $\theta^{(2)}$, and the wave number is $k^{(2)}$. The refraction angle of the refracted $\mathrm{SV}$-wave is $\theta^{(4)}$, and the wave number is $k^{(4)}$. The reflection coefficient of P-P wave is R1, the reflection coefficient of $\mathrm{P}-\mathrm{SV}$ wave is $\mathrm{R} 3$, the refraction coefficient of P-P wave is R2, and the refraction coefficient of P-SV wave is R4. Here, $\theta^{(0)}=\theta^{(1)}=\theta, k^{(0)}=k^{(1)}=k, P^{(0)}$, $P^{(1)}, P^{(2)}, S V^{(3)}$ and $S V^{(4)}$ are particle displacements of incident, reflected and refracted $\mathrm{P}$-waves and reflected and refracted SV-waves, respectively. Superscripts 0, 1, 2, 3 and 4 indicates incident $\mathrm{P}$-waves, reflected $\mathrm{P}$-waves, refracted $\mathrm{P}$ waves, reflected SV-waves and refracted SV-waves, respectively [9].

The normalized expressions of the particle displacements of various modes converted by incident $\mathrm{P}$-wave at the interface can be written as the following equations [10].

$$
\begin{aligned}
& P^{(0)}=\left\lfloor\begin{array}{c}
\sin \theta \\
-\cos \theta
\end{array}\right] \exp [j(\omega t-k x \sin \theta+k z \cos \theta)] \\
& P^{(1)}=R_{1}\left[\begin{array}{c}
\sin \theta \\
\cos \theta
\end{array}\right\rfloor \exp [j(\omega t-k x \sin \theta-k z \cos \theta)]
\end{aligned}
$$

$$
\begin{aligned}
& S V^{(3)}=R_{3}\left[\begin{array}{c}
\cos \theta^{(3)} \\
\sin \theta
\end{array}\right] \exp \left[j\left(\omega t-k^{(3)} x \sin \theta^{(3)}-k^{(3)} z \cos \theta^{(3)}\right)\right] \\
& P^{(2)}=R_{2}\left[\begin{array}{c}
\sin \theta^{(2)} \\
-\cos \theta^{(2)}
\end{array}\right] \exp \left[j\left(\omega t-k^{(2)} x \sin \theta^{(2)}+k^{(2)} z \cos \theta^{(2)}\right)\right] \\
& S V^{(4)}=R_{4}\left[\begin{array}{c}
\cos \theta^{(4)} \\
\sin \theta^{(4)}
\end{array}\right] \exp \left[j\left(\omega t-k^{(4)} x \sin \theta^{(4)}+k^{(3)} z \cos \theta^{(4)}\right)\right]
\end{aligned}
$$

The results show that the stress components of the incident $\mathrm{P}$-wave and mode converted wave $\mathrm{T} 3$ related to the boundary conditions at zero time and reflection point are as follows.

$$
\left[\begin{array}{c}
T_{3}^{(0)} \\
T_{3}^{(1)} \\
T_{3}^{(2)} \\
T_{3}^{(3)} \\
T_{3}^{(4)}
\end{array}\right]=\left[\begin{array}{c}
-j k L_{0} \\
-j k \mathrm{R}_{1} L_{1} \\
-j k^{(2)} R_{2} L_{2} \\
-j k^{(3)} R_{3} L_{3} \\
-j k^{(4)} R_{4} L_{4}
\end{array}\right]
$$

Among them, LO L4 represent the sum of the vector relations of the elastic modulus corresponding to $\mathrm{P}$-waves and SV-waves in the direction of $T 3$ stress component through different incident and reflective media in the case of incident and refraction.

The stress component of $\mathrm{T} 3$ is as follows.

$$
\left[\begin{array}{c}
T_{5}^{(0)} \\
T_{5}^{(1)} \\
T_{5}^{(2)} \\
T_{5}^{(3)} \\
T_{5}^{(4)}
\end{array}\right]=\left[\begin{array}{c}
j k M_{0} \\
-j k R_{1} M_{1} \\
j k^{(2)} R_{2} M_{2} \\
j k^{(3)} R_{3} M_{3} \\
j k^{(4)} R_{4} C_{44}^{\prime} M_{4}
\end{array}\right]
$$

Among them, MO M4 are the sum of the vector relations of the elastic modulus of P-waves and SV-waves in the direction of $T 5$ stress component through different incident and reflective media under the condition of incident and refraction.

From the continuous boundary condition of particle displacement on the interface of medium, we can get the following results.

$$
\begin{aligned}
& P_{x}^{(0)}+P_{x}^{(1)}+S V_{x}^{(3)}=P_{x}^{(2)}+S V_{x}^{(4)} \\
& P_{z}^{(0)}+P_{z}^{(1)}+S V_{z}^{(3)}=P_{z}^{(2)}+S V_{z}^{(4)}
\end{aligned}
$$

Where $P_{x}$ and $P_{z}$ represent the components in the $\mathrm{x}$ and $\mathrm{z}$ directions of the $\mathrm{P}$ wave, respectively. $S V_{x}$ and $S V_{z}$ represent the components in the $\mathrm{x}$ and $\mathrm{z}$ directions of the $\mathrm{SV}$ wave, 
respectively.

According to the constitutive equation of elastic solid medium $T=C: S$ ( $T$ is the stress and $C$ is the modulus of elasticity) [11], the stress components of mode conversion waves $T 3$ and $T 5$ at 0 time can be obtained as follows.

$$
\begin{aligned}
& T_{3}^{(0)}+T_{3}^{(1)}+T_{3}^{(2)}=T_{3}^{(2)}+T_{3}^{(4)} \\
& T_{5}^{(0)}+T_{5}^{(1)}+T_{5}^{(3)}=T_{5}^{(2)}+T_{5}^{(4)}
\end{aligned}
$$

Since the velocity and density of P-wave and SV-wave in the incident and refracted media are known, when the incident angle of $\mathrm{P}$-wave is given, the refraction angle of each mode conversion can be obtained by Snell ' s law.

The refractive and reflective coefficients of the interface are related to $\sin \theta, \cos \theta, k, L O \sim L 4$ and $M O \sim M 4$ through equations (8) - (18). When the given quantities are known, they can be substituted into the above formula and solved by simultaneous equations. At this time, the calculation of refraction and reflection coefficient can be further simplified and analyzed, which can be used as a reference for the next simulation.

\section{THE ESTABLISHMENT OF FINITE ELEMENT SIMULATION MODEL}

The signal source emits a sweeping single-tone sine wave that travels to the target and is reflected, and a string of geophones on the ground measure the speed of surface vibration [12]. Given the propagation rate of waves in a material, the time delay can be used between signal transmission and reception at the surface to obtain an estimate of the distance to the target. This simulation mainly adopts the solid mechanical physical field of COMSOL Multiphysics for design, and the research is transient. COMSOL Multiphysics is a large advanced numerical simulation software, which is widely used in scientific research and engineering calculation in various fields [13]. It is called the first real multi-physics direct coupling analysis software by scientists in the world.

\section{A. The establishment of different PE pipe diameter simulation model.}

The simulation model established by COMSOL is shown in Fig.1

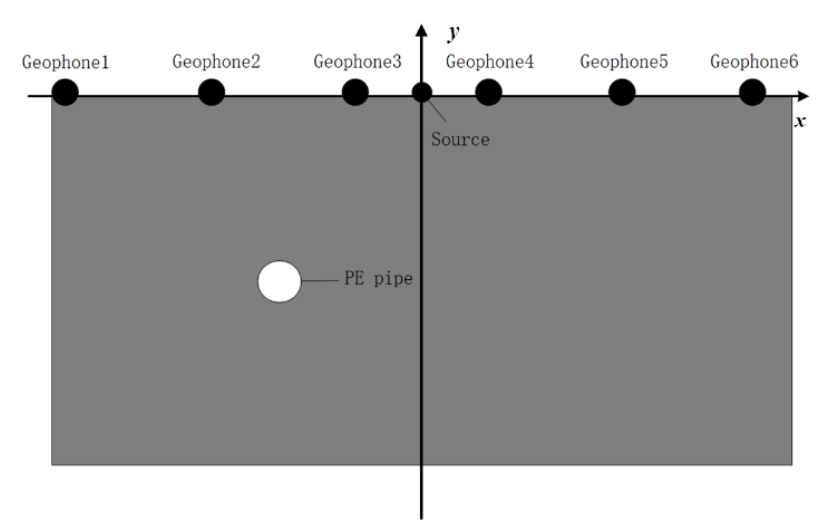

Fig.1 Simulation models of different PE tube diameters

The physical field used in this experiment is mainly solid mechanics, and the study is transient. The physical field only selects other fields except the above seven rectangles [14]. The boundary load type is the total force, and its additional range is the lower boundary of the signal transmitter, namely the lower edge of the fourth rectangle. The buried PE pipe wall is set as a fixed constraint, and the other boundaries are set as free [15]. The expression is an analytical function defined in the parameter setting, that is, an $1(\mathrm{t})=\mathrm{s}(\mathrm{t})$. In the global definition, the parameters are defined and the signal source function is set (the signal period emitted by the signal source is $\mathrm{T}_{0}=60 \mathrm{~s}$ ), the initial tube depth is set to $4 \mathrm{~m}$, and the signal source adopts sinusoidal scanning signal, whose frequency is $500 \mathrm{~Hz}$ to $1000 \mathrm{~Hz}$, and the duration is one minute. The scanning sine function can be expressed as:

$$
\operatorname{sweep}(\mathrm{t})=\sin \left(2 * \mathrm{pi}^{*} \mathrm{f} 0\left(\mathrm{k}^{t}-1\right) / \log (\mathrm{k})\right)
$$

where $k=\exp (\log (\mathrm{f} 1 / \mathrm{f} 0) / \mathrm{t} 0)$, so the emission signal used in this experiment is $s(\mathrm{t})=\sin \left(1000 * \mathrm{pi}^{*}\left(1.005^{t}-1\right) / 0.0022\right)$. Signal source function diagram as shown in.Fig.2.

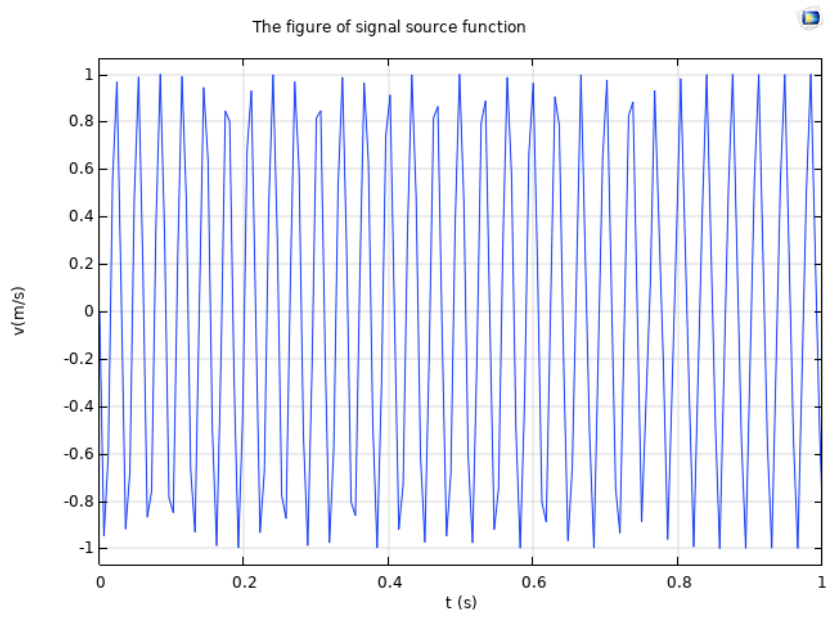

Fig. 1 The figure of signal source function 
For the setting of relevant parameters of geometric materials, steel AISI 4340 is set for the tube, and other domain materials are set as empty materials. Different Poisson's ratio and elastic modulus E are set for different soils (P-wave speed is $\sqrt{(\mathrm{E} / \rho)})$. Three different soils were used in this experiment. (1) oil density $\rho=2200 \mathrm{~kg} / \mathrm{m}^{3}$, Poisson's ratio $v=0.25$, elastic modulus $E=16 \mathrm{MN} / \mathrm{m}^{2}$, $V_{p}=85.28 \mathrm{~m} / \mathrm{s}$; (2) Clay(hard state): $\rho=1900 \mathrm{~kg} / \mathrm{m}^{3}, V=0.25$, $E=1.25 * 10^{7} \mathrm{~Pa}, V_{p}=81.11 \mathrm{~m} / \mathrm{s}$; (3) Sandy clay: $V=0.36$, $E=3.5 * 10^{7} \mathrm{~Pa}$; The clay $\rho=1.8-2.0 \mathrm{~kg} / \mathrm{cm}^{3}, \quad V_{P}=98.46 \mathrm{~m} / \mathrm{s}$. When simulating different soils, the specific operation can be directly carried out by changing these parameters [16].

Finally, in the setting of "study", because this experiment needs to study different buried depths and the size of buried $\mathrm{PE}$ pipe, if a set of parameters are very troublesome to modify, it is necessary to use parametric scanning (as shown in Table 1.).

Table 1. Size Parameterized Scanning Setting of Buried PE Pipe

\begin{tabular}{|c|c|c|}
\hline $\begin{array}{c}\text { parameter } \\
\text { name }\end{array}$ & $\begin{array}{c}\text { List of parameter } \\
\text { values }\end{array}$ & $\begin{array}{c}\text { Parameter } \\
\text { units }\end{array}$ \\
\hline $\mathrm{d}$ & Range $(7.5,2.5,15)$ & $\mathrm{mm}$ \\
\hline
\end{tabular}

Transient setting range $(0, \mathrm{~T} 0 / 40,10 * \mathrm{~T} 0)$.

\section{B. Establishment of simulation model of different PE pipe buried depth}

The setting of relevant parameters of geometric materials is the same as those in Table 1 and Table 2 in Section A of chapter 3 , so it is not introduced in detail here. Due to the different buried depth of PE pipe, it is necessary to add a parametric scanning in the process of simulation "research". The following Table 2 shows:

Table 2. Parameterized Scanning Setting of Pipe Buried Depth

\begin{tabular}{|c|l|c|}
\hline parameter name & $\begin{array}{l}\text { List of parameter } \\
\text { values }\end{array}$ & parameter units \\
\hline $\mathrm{dsp}$ & Range $(-1,-1,-4)$ & $\mathrm{m}$ \\
\hline
\end{tabular}

' Transient ' setting range is $(0, \mathrm{~T} 0 / 40,10 * \mathrm{~T} 0)$.

\section{THE Modeling and Simulation of Different Soil Medium}

The simulation model established by COMSOL Multiphysics is shown in Fig. 3 as follows, due to the mixed soil, the relevant parameters of the upper soil are soil density $\rho=2200 \mathrm{~kg} / \mathrm{m}^{3}$, Poisson 's ratio $v=0.25$, elastic modulus
$E=16 M N / m^{2}, v_{p}=85.28 \mathrm{~m} / \mathrm{s}$, height $2 \mathrm{~m}$, and the lower soil is clay (hard state): $\rho=1900 \mathrm{~kg} / \mathrm{m}^{3}, v=0.25, \quad E=1.25^{*} 10^{7} \mathrm{~Pa}$, $v_{p}=81.11 \mathrm{~m} / \mathrm{s}$, soil depth is $2.5 \mathrm{~m}$ [17]

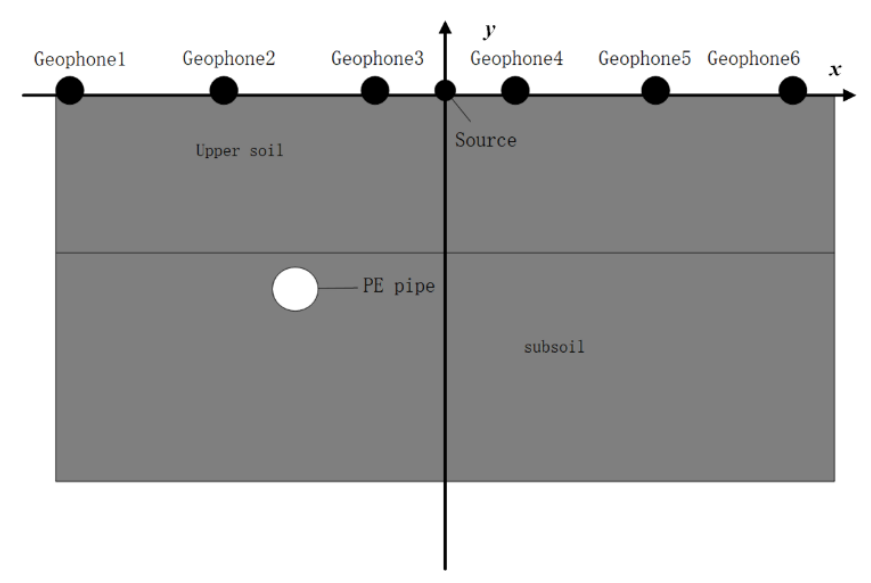

Fig.2 Simulation models of different soil media

Table 3 Parameterized Scanning Setting of Pipe Buried Depth

\begin{tabular}{|c|c|c|}
\hline parameter name & $\begin{array}{c}\text { List of parameter } \\
\text { values }\end{array}$ & parameter units \\
\hline $\mathrm{dsp}$ & Range $(-1,-1,-4)$ & $\mathrm{m}$ \\
\hline
\end{tabular}

The settings of other geometric material parameters are the same as those in the previous sections, so no recapitulation is made.

\section{SIMULATION RESULTS ANALYSIS}

In the estimation of target distance, when the position of signal transmitter and geophone overlaps, the distance between signal transmitter and buried PE pipe can be obtained by the obtained propagation time. Then, two points with the same time of received signal are obtained by moving the position of the detector, and their distance from the buried PE pipe is approximately equal. At this time, the distance between the midpoint of the two points and the signal transmitter is known, plus the distance between the signal transmitter and the buried PE pipe, and the estimation of the target distance can be roughly obtained through the Pythagorean theorem.

The simulation is divided into three parts, which analyzes and studies the applicability of the positioning algorithm to different PE pipe radius, different buried PE pipes and different soil media [18].

In this simulation, the PE pipe is located at an initial position of $(7 \mathrm{~m},-4 \mathrm{~m})$ and the pipe has a straight radius of $0.2 \mathrm{~m}$, so the point reflector position is $(7 \mathrm{~m},-3.9 \mathrm{~m})$. The 
position of the signal transmitter (taking the its lower boundary as the center) is $(10.3 \mathrm{~m}, 0)$ and the location of the first geophone (also taking its lower boundary as the center) is $(0.15 \mathrm{~m}, 0)$, while the remaining five geophones are placed with an interval of 4 meters one next to another. When analyzing the data of different pipe burial depths, the first geophone (i.e. bnd1) is taken as the main research object. The delay characteristics of the reflected signal at the same depth are mainly examined at a depth of $4 \mathrm{~m}$ [19]. The time required for the six geophones to receive the reflected signal is obtained respectively.

Geometric knowledge indicates that for the ith geophone, the time required by the transmission of the input signal at the distance $x i$ for the point reflector at any location $(x, z)$ is:

$$
t=\frac{\sqrt{\left(\left(\mathrm{x}-\mathrm{x}_{s}\right)^{2}+z^{2}\right)}+\sqrt{\left(\left(\mathrm{x}-\mathrm{x}_{i}\right)^{2}+\mathrm{z}^{2}\right)}}{c}
$$

where $x_{i}=D *(\mathrm{i}-1)+\mathrm{h} / 2, h$ is the geophone width,

$i$ is $1,2, \ldots$ N. $c$ is the shear wave speed, $c=\sqrt{(\mathrm{C} 11 / \rho)}$, $C_{l l}$ is the elastic modulus E of soil, and $\rho$ is the density of soil.

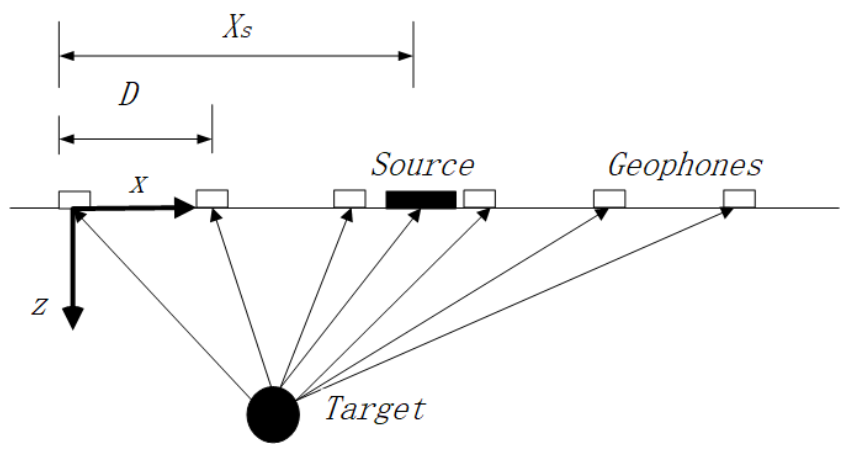

Fig 4. Schematic diagram of receiving and transmitting signals

\section{A. Effect of buried PE pipe depth on the received signal of geophone}

In this case, the soil density is taken from $\rho$ to $2200 \mathrm{~kg} / \mathrm{m}^{3}$, Poisson's ratio is 0.25 , elastic modulus is $16 \mathrm{MN} / \mathrm{m}^{2}$, and $c$ is $85.28 \mathrm{~m} / \mathrm{s}$. At the depth of 4 meters, the time used by the signal traveling from source to geophones is shown in Table 1 .

Table 4. Time used by signal travelling from source to geophones

\begin{tabular}{|l|l|l|l|l|l|l|}
\hline $\begin{array}{l}\text { Num } \\
\text { ber }\end{array}$ & 1 & 2 & 3 & 4 & 5 & 6 \\
\hline
\end{tabular}

\begin{tabular}{|c|l|c|c|c|c|c|}
\hline Tim & 0.152 & 0.116 & 0.107 & 0.135 & 0.176 & 0.220 \\
$\mathrm{e}$ & $34 \mathrm{~s}$ & $55 \mathrm{~s}$ & $58 \mathrm{~s}$ & $66 \mathrm{~s}$ & $54 \mathrm{~s}$ & $74 \mathrm{~s}$ \\
\hline
\end{tabular}

As can be calculated, the signal delay $t_{2 \rightarrow 3}$ is $0.00897 \mathrm{~s}$, $t_{4 \rightarrow 2}$ is $0.01911 \mathrm{~s}, t_{l \rightarrow 4}$ is $0.01668, t_{5 \rightarrow 1}$ is $0.0242 \mathrm{~s}$, and $t_{6 \rightarrow 5}$ is 0.0442 . The calculated data combined with figure 4 can be drawn: the shortest time for No.3 seismic geophone to receive signals is due to its nearest distance to the transmitter and the target PE tube, while the longest time for No.6 seismic geophone to receive signals is due to its farthest distance to the transmitter and the target PE tube. When the depth is certain, the global map of the speed distribution of the signal reflected by the buried PE pipe received by each geophone on the surface is shown in Fig.5 PE pipe.

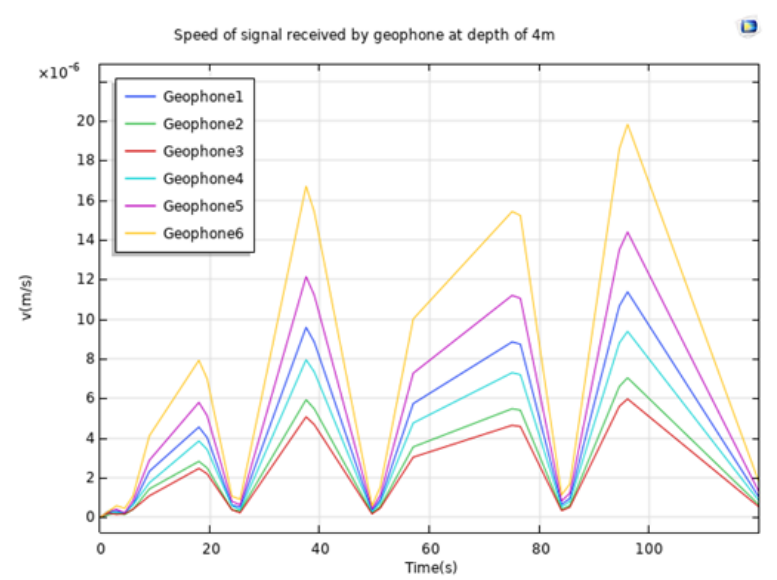

Fig. 5 Speed of signal received by geophones at a depth of $4 \mathrm{~m}$

For the first geophone [20], the time used the received signal from emission to reflection when the buried PE pipe is at different depths is shown in Table 2 as follows.

Table 5. Time used by the signal travelling from source to receiver when the PE pipe is buried at different depths

\begin{tabular}{|c|c|c|c|c|}
\hline Depth & $1 \mathrm{~m}$ & $2 \mathrm{~m}$ & $3 \mathrm{~m}$ & $4 \mathrm{~m}$ \\
\hline Time & $0.12112 \mathrm{~s}$ & $0.12801 \mathrm{~s}$ & $0.13874 \mathrm{~s}$ & $0.15234 \mathrm{~s}$ \\
\hline
\end{tabular}

Similarly, the signal delay: $t_{2 m \rightarrow 1 m}$ is $0.00689 \mathrm{~s}, t_{3 m \rightarrow 2 m}$ is $0.01073 \mathrm{~s}, t_{4 m \rightarrow 3 m} \mathrm{~s}$ is $0.0136 \mathrm{~s}$. According to the calculation results, the delays are tiny.

When the buried depth of the tube is different, the global shape of the reflected signal received by the same detector is roughly the same, and there is a certain delay between them. The calculation shows that their delay is small, so the reaction is not obvious.

\section{$B$. The influence of buried PE pipe size on the signal received by geophone at certain depth PE pipe}


When the buried depth is certain, the time required by the PE pipes of different thicknesses to reflect the signal to the 1st geophone is shown in Fig.3 below. This can be divided into two cases: variable internal radius caused by different pipe wall thicknesses with fixed external radius of the PE pipe, and variable external radius caused by different pipe wall thicknesses with fixed internal radius. $d / 2$ indicates the difference between the external and internal radius of the pipe; the soil density is $2200 \mathrm{~kg} / \mathrm{m}^{3}$; Poisson's ratio is 0.25 ; elastic modulus $E$ is $16 \mathrm{MN} / \mathrm{m}^{2}$. According to the formula, $c$ is $85.28 \mathrm{~m} / \mathrm{s}$ [21], [22].

(1) variable internal radius caused by different wall thicknesses with certain external radius.

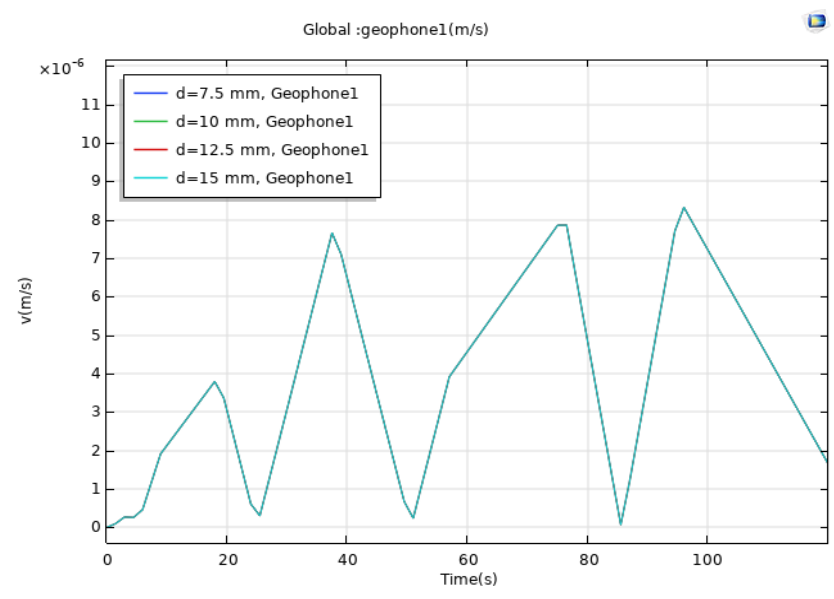

Fig. 6 Speed of the signal received by the geophone 1 with variable internal radius and certain buried depth and external radius

As shown in the Fig.6, if the external radius of the pipe is certain, at the buried depth of $1 \mathrm{~m}$, the variable wall thickness has no influence on the time required for the signal to propagate to the receiver. The reason for this is in this simulation the signal generated by the source is reflected by the pipeline external wall, and when the depth of the external wall is unchanged, the speed of the reflected signal and the time used should be the same. This can also be well explained by Fig. 6 .

(2) Variable external radius caused by different pipe wall thicknesses with certain internal pipe radius.

Table 6 Time used by the signal from source to geophone 1 with variable external radius and certain buried depth and internal radius.

\begin{tabular}{|c|c|c|c|c|}
\hline $\begin{array}{l}\text { The } \\
\text { thickness of } \\
\text { PE pipe: } \mathrm{d} / 2\end{array}$ & $\mathrm{~d}=7.5$ & $\mathrm{~d}=10$ & $\mathrm{~d}=12.5$ & $\mathrm{~d}=15$ \\
\hline Time & $0.12113 \mathrm{~s}$ & $0.12112 \mathrm{~s}$ & $0.12111 \mathrm{~s}$ & $0.121111 \mathrm{~s}$ \\
\hline
\end{tabular}

The buried depth of the pipeline is $1 \mathrm{~m}$. When the external radius of the pipeline changes, the time required for the geophone 1 to receive the signal varies with it [23]. When the internal radius is fixed, and the wall thickness increases, the distance covered by the signal from the source and reflected by the PE pipeline will be shortened, thereby reducing the time consumed. Since the difference between the internal and external radius is small, the time difference obtained according to the equation is also small. Therefore, at different pipeline thicknesses, the delay of the reflected signal received by the geophone is also tiny.

\section{Signal received by the geophone when the PE pipe is buried in mixed soil}

The time required for the geophone on the ground to receive the signal when the $\mathrm{PE}$ pipe is in the mixed soil is analyzed. In this case, the external radius of the pipe is $100 \mathrm{~mm}$, and the thickness of the pipe wall is $5 \mathrm{~mm}$. The surface is divided into two layers: the upper layer has the density of the upper soil is $2200 \mathrm{~kg} / \mathrm{m}^{3}$, Poisson's ratio is 0.25 , elastic modulus $\mathrm{E}=16 \mathrm{MN} / \mathrm{m}^{2}$, c is $85.28 \mathrm{~m} / \mathrm{s}$ according to the equation 2 and the thickness is $2 \mathrm{~m}$; the lower layer at hard state has Poisson's ratio of $0.25, E$ of $1.25^{*} 10^{7}$ Pas, $c$ of $81.11 \mathrm{~m} / \mathrm{s}, \rho$ of $1900 \mathrm{~kg} / \mathrm{m}^{3}$, and the thickness of the lower layer of $2.5 \mathrm{~m}$, respectively [24]. The experiments were implemented in three different cases: first, the PE pipe is in the upper soil, second, the PE pipe is partly located in the upper soil, partly in the lower soil, and third, the PE pipe is all in the lower soil. (The depth is $1 \mathrm{~m}$ in the first case, $2 \mathrm{~m}$ depth in the second case, and $3 \mathrm{~m}$ or $4 \mathrm{~m}$ in the third case.) The following equations are used to solve the time used by the signals from the source to geophone a depth of 3 or 4 meters [5].

$$
\left.\begin{array}{l}
\frac{H_{1}}{v_{p}^{(1)} \cos \theta_{1}}+\frac{H_{2}}{v_{p}^{(2)} \cos \theta_{2}}=\frac{t}{2} ; \\
\frac{\sin \theta_{1}}{v_{p}^{(1)}}=\frac{\sin \theta_{2}}{v_{p}^{(2)}} ; \\
S_{1}+S_{2}=7 ; \\
S_{1}=H_{1} \tan \theta_{1}, S_{2}=H_{2} \tan \theta_{2}
\end{array}\right\}
$$

Table 7. Time used by geophone 1 to receive the signal at different depth of burial in mixed soil

\begin{tabular}{|c|c|c|c|c|}
\hline $\begin{array}{c}\text { Dept } \\
\mathrm{h}\end{array}$ & $1 \mathrm{~m}$ & $2 \mathrm{~m}$ & $3 \mathrm{~m}$ & $4 \mathrm{~m}$ \\
\hline
\end{tabular}




\begin{tabular}{|c|c|c|c|c|}
\hline \multirow{2}{*}{ Time } & 0.121123 & 0.128008 & 0.176996 & 0.189308 \\
& $\mathrm{~s}$ & $\mathrm{~s}$ & $\mathrm{~s}$ & $\mathrm{~s}$ \\
\hline
\end{tabular}

Because it is mixed soil, the propagation rates of waves are different at different layers [25]. When the PE pipe is in the upper soil (the pipe is buried at a depth of $1 \mathrm{~m}$ ), the propagation time is the same as the previous conclusion. When the PE pipe is between two layers of soil (the buried depth of the pipe is $2 \mathrm{~m}$ ) and the reflection point is still in the upper layer of soil $(c=85.28 \mathrm{~m} / \mathrm{s})$, the propagation time is the same as the previous conclusion. When the buried PE pipe is totally located in the lower soil (the pipe is buried at a depth of $3 \mathrm{~m}$ or $4 \mathrm{~m}$ ), due to the different speed of sound wave propagation in different soil, the time used is longer than that in the same depth of the buried PE pipe in a single soil. In this simulation study, the propagation velocity in the upper soil is $85.28 \mathrm{~m} / \mathrm{s}$, and that in the lower soil is $81.11 \mathrm{~m} / \mathrm{s}$. Therefore, the sound wave is transmitted from the upper soil to the lower soil, the speed is slowed down, and the time required is prolonged.

\section{CONCLUISIONS}

In this paper, the acoustic detection technology of buried PE pipe is studied, the simulation model is established, and the theoretical basis algorithm based on principle is calculated and verified. The main contents of this study are as follows:

(1) The propagation characteristics of sound waves have a great influence on locating of buried PE pipes. The receiver can receive two waveforms: surface wave generated by the ground and reflected wave by the PE pipe. The buried PE pipe can be located according to the derived equations and analysis on the waveform.

(2) Difference of time between signal reflection and different geophone are small when buried depth is different.

(3) When the buried depth is constant, and the outer diameter of the PE pipe is constant, the internal diameter changes with the thickness of the pipe wall, and the time taken by the geophone to receive the signal is constant. When the buried depth is constant and the internal diameter is constant, the external diameter changes with the wall thickness. Because the wall thickness is small, the delay of the geophone received signal is small.

(4) When the buried PE pipe is in the mixed soil, when it is located between the upper soil or two layers of soil, the signal travelling time is the same as when the buried pe pipe is in the single layer soil. When it is in the lower soil, the time taken by the geophone to receive the signal increases with the that at the same depth of the single layer soil.

The limitation of this study lies in the need to make the approximate range of buried PE pipe clear before detection, and requirements for land and pipeline. At the same time, the simulation is in the ideal situation and has difference from the reality. In the future research, its limitations should be solved as far as possible. At the same time, attention should be paid to various factors in the reality, so as to develop in a more intelligent direction.

\section{Acknowledgments}

This work is supported by the International Science and Technology Cooperation and Exchange Research Project of Sichuan Province(18GJHZ0195), the International Science and Technology Cooperation Project of Chengdu(2020GH02-00016-HZ), Research on the shallow medium dynamic model and imaging algorithm based on the elastic wave reflection method based on the open fund project of the National Mountain Highway Engineering Technology Research Center (GSGZJ-2020-01) ,Downhole Intelligent Measurement and Control Science and Technology Innovation Team of Southwest Petroleum University(2018CXTD04), and the National Natural Science Foundation(51974273).

\section{References}

[1] Zhang S.X. Talk about the application of APL acoustic pipeline locators in urban underground non-metallic pipeline detection[J]. Low Carbon World, 2015 (29): 241-242.

[2] Cheng S , Cai Z . Simulation analysis of multi physical field coupling based on COMSOL $[\mathrm{J}]$. Northeast Electric Power Technology, 2017.

[3]Li K.J., Ye Z.H., Shen Y.G., Xu J.J. .Leakage detection of water supply pipeline based on ground penetrating radar technology under complex overburden pavement[J].Scientific and technological communication. 2021, 37 ( 03 ) $81-85$

[4] Wang C.; Liu Z.G.; Li M.F.; S F.; Zhang G.X. AcousticsSeismic Coupling Mine Technology Analysis,[J]. Journal of Acoustics, 2008, 33 (4): 354-359

[5] Xiao L.W.; Jia Z.X.; L Y.J. Buried Non-Metallic (PE) 
Pipeline Detection New Technology[J]. Mapping Advisory, 2013 (S2): 126-127.

[6] Dvnh A, Ab A, Hnnm A, et al. Investigation of thermal residual stresses during laser ablation of tantalum carbide coated graphite substrates using micro-Raman spectroscopy and COMSOL multiphysics - ScienceDirect[J]. Ceramics International, 2020, 47(3):3498-3513.

[7] Wu W., Zhang G.Q., Yi S., Sun H.B., Wang P.D., Zhang Y.J.. Study on propagation characteristics of acoustic emission signal in gas pipeline[J].Science and technology winds 2021, ( 09 ) 85-86.

[8] Li B L , Fang X Q , Zhang T F, et al. Elastic-slip interface effect on dynamic response of underwater convey tunnel in saturated poroelastic soil subjected to plane waves $[\mathrm{J}]$. Tunnelling and Underground Space Technology, 2020, 103:103468.

[9] Fa L.; Lu X.; Zheng Wen Zeng; Liu Y.M.; Li G.H. Transmission network model of seismically reflected signals in elastic stratum media[J]. Oil Instruments, 2010, 24 (1): 40-46.

[10] L.Fa, R. L.Brown and J. P. Cast ag na.Anomalous post critical refraction behavior for certain transversely isotropic media[J].J.Acoust.Soc.120(2006).

[11] Wang E , Ba J , Carcione J M , et al. Effect of local fluid flow on the reflection and transmission of elastic waves at an interface between an elastic solid and a double-porosity medium[J]. Geophysics, 2020, 85(4):1-60.

[12] Cheng F , Liu J , Jing W, et al. Locating Leaking Buried Pipes Based on Ground Microseismic Records in 3D Space[J]. Surveys in Geophysics, 2018, 39:9931007.

[13] Latif M , Sanei S , Chambers J , et al. Localization of abnormal EEG sources using blind source separation partially constrained by the locations of known sources[J]. IEEE Signal Processing Letters, 2006, 13(3):117-120.

[14] Gulino M S , Bruzzi M , D Vangi. Gas-Coupled Laser Beam Detection technique for NDT of mechanical components[J]. Ultrasonics, 2021.

[15] Sheil B , Martin C, Byrne B . Simulation of overburden pressure during laboratory investigations of axial pipe-soil interaction[J]. Géotechnique, 2019.

[16] Zhang Z P , Shao J Q , Sun X Y, et al. Simulation of Soil Water and Salt Transport with Sand Column in Coastal Saline Soil Based on COMSOL[J]. Applied Mechanics and Materials, 2014, 614:668-671.

[17] Ren C.J., An W.D., Yang J. .Discussion on Key Technology of Small Diameter Pipeline Detection and Location $[\mathrm{J}]$.Automation and instrumentation. 2021, ( 03 ) 188 - 191.

[18] Zhang P.P. Principle research and implementation of acoustic directional transmitter[J].Digital communication world. 2019, (05) $146+273$.

[19] Y Hou, Jiao R, Yu H . MEMS based geophones and seismometers - ScienceDirect[J]. Sensors and Actuators A: Physical, 2020, 318.

[20] Y Wang, N Fu, Fu Z, et al. A Semi-Automatic Coupling Geophone for Tunnel Seismic Detection[J]. Sensors, 2019, 19(17):3734-.

[21] Peng Y.M. Application of acoustic detection in PE gas pipeline $[\mathrm{J}]$. Seminar on Gas Operation and Safety in China (tenth session) and Papers of 2019 Academic Annual Meeting of Gas Branch of Chinese Society of Civil Engineering (Volume II) 236 - 237.

[22] Fu Y. Ultrasonic detection of polyethylene (PE) tube longitudinal wave single oblique probe[J]. Safety of special equipment in China. 2011, 27 (09)

[23] Hansen S M , Schmandt B . Automated Detection and Location of Microseismicity at Mount St. Helens with a Large-N Geophone Array[J]. Geophysical Research Letters, 2015, 42(18):7390-7397.

[24] Cheng X.S. Mixed use analysis of different types of seismic geophones[J]. Geophysical prospecting equipment. 2020, 30 (05) $281-283+288$.

[25] Nujid M M , Taha M R . Assessment on Bearing Capacity of Two Clay Layers Soil Using COMSOL[J]. Applied Mechanics and Materials, 2014, 567:675-680.

\section{Creative Commons Attribution License 4.0 (Attribution 4.0 International, CC BY 4.0)}

This article is published under the terms of the Creative Commons Attribution License 4.0 https://creativecommons.org/licenses/by/4.0/deed.en_US 